\title{
Maladaptive Proximal Tubule Repair: Cell Cycle Arrest
}

\author{
Joseph V. Bonventre
}

Renal Division and Biomedical Engineering Division, Department of Medicine, Brigham and Women's Hospital, Harvard Medical School, Boston, Mass., and Division of Health Sciences and Technology, Harvard-Massachusetts Institute of Technology, and Harvard Stem Cell Institute, Cambridge, Mass., USA

\section{Key Words}

Acute kidney injury · Chronic kidney disease · Kidney aging · Senescence

\begin{abstract}
Acute kidney injury (AKI) leads to worsening of chronic kidney disease (CKD), and CKD predisposes to the clinical entity of AKI. The tubules of the kidney play a central role in the fibrotic response, which ultimately leads to progressive kidney disease. The cellular mechanisms responsible for the epidemiological association between AKI and CKD are complex. In order to unravel characteristics of this direct involvement of the tubules, in particular the proximal tubules, we established a model to specifically target injury to the proximal tubule using a genetic approach to express the simian diphtheria toxin (DT) receptor in the proximal tubule. A single administration of DT to the proximal tubule resulted in inflammation, reversible injury, and adaptive repair. By contrast, thrice repeated injury led to maladaptive repair with sustained tubule injury, vascular rarefaction, proliferation of interstitial myofibroblasts, interstitial fibrosis, and glomerular sclerosis. An important feature of the maladaptive repair process after severe injury is the development of cell cycle arrest in G2/M. There is a subsequent activation of the DNA repair response with activation of a secretory phe-
\end{abstract}

notype whereby profibrotic factors are released. This insight introduces a number of potential new targets for therapeutic intervention to prevent and/or arrest CKD progression.

๑) 2014 S. Karger AG, Basel

\section{Introduction}

There is strong epidemiological evidence of a causal clinical connection between the clinical syndrome of acute kidney disease (AKI) and the subsequent development of chronic kidney disease (CKD) $[1,2]$. AKI leads to worsening of $\mathrm{CKD}$ and $\mathrm{CKD}$ predisposes to the clinical entity of AKI. The tubules of the kidney play a central role in the fibrotic response, which ultimately leads to progressive kidney disease [3-5]. The cellular mechanisms responsible for the epidemiological association between $\mathrm{AKI}$ and CKD are complex. Chronic processes that originate in the glomerulus or the tubulointerstitium lead to interstitial fibrosis and tubular atrophy. Once the process is initiated, it often persists even if the initiating factors or

Targeting Recovery from Acute Kidney Injury: Round Table Conference at the 19th International Conference on Continuous Renal Replacement Therapies (Manchester Grand Hyatt, San Diego, Calif., USA, March 2-3, 2014).

\section{KARGER}

E-Mail karger@karger.com

www.karger.com/nec
(C) 2014 S. Karger AG, Basel

1660-2110/14/1274-0061\$39.50/0
Joseph V. Bonventre, $\mathrm{MD}, \mathrm{PhD}$

Renal Division, Brigham and Women's Hospital

Harvard Medical School

Boston, MA 02115 (USA)

E-Mail joseph_bonventre@hms.harvard.edu 
disease process is no longer operant. Basile et al. [6] reported vascular dropout after ischemic injury in the rodent. We found residual protection of the mouse kidney against a subsequent ischemic insult even 6-12 weeks after ischemia reflecting the fact that there were persistent changes in the kidney even though these had generally been underestimated in the past. When the kidney was examined over time after injury, we found fibrosis that developed over time even when the initial insult was selflimited $[7,8]$.

Injury to the glomerulus and the development of glomerulosclerosis is often taken as evidence of primary glomerular disease. It is known, however, that ischemia can lead to glomerular pathology [9]; thus, disease that originates in the tubules and interstitium, which leads to vascular compromise, may secondarily affect the glomerulus, as we describe in this review. Here, we focus on the mechanisms of injury to the tubule that can result in interstitial fibrosis and glomerulosclerosis.

\section{How Do the Tubules Respond to Specific Focal Lesions of Epithelial Cells?}

We have generated bigenic Six2-GFPCre+;iDTR+ $\left(\mathrm{DTR}^{\mathrm{rec}}\right)$ mice where the simian diphtheria toxin (DT) receptor was incorporated in renal epithelial cells (rec) that derived from the metanephric mesenchyme [10]. This made the proximal tubule particularly susceptible to injury from intravenous administration of DT. A single low sublethal dose of DT resulted in a vigorous inflammatory response with upregulation of intercellular adhesion molecule-1 expression and likely other endothelial adhesion molecules, with attraction of neutrophils, macrophages, and T lymphocytes to the kidney. This was accompanied by a focal moderate increase in cell numbers in the tubulointerstitium. There was an adaptive proliferative response of surviving proximal tubule epithelial cells and the inflammatory process spontaneously resolved with very few sequelae.

By contrast, after three doses of DT, administered at weekly intervals, there was maladaptive repair with persistence of a chronic interstitial infiltrate, increased myofibroblast proliferation, tubulointerstitial fibrosis, and tubular atrophy as well as an increase in serum creatinine compared to controls $(0.6 \pm 0.1$ vs. $0.18 \pm 0.02 \mathrm{mg} / \mathrm{dl})$ by week 5, 2 weeks after the last dose in the thrice-treated animals. The number of interstitial cells which stained positively with antibodies to the platelet-derived growth factor receptor $\beta+$ (pericytes/perivascular fibroblasts) was also increased as were aSMA+ (myofibroblasts), FSP-1 (fibroblast-specific protein-1)/S100A4+ (fibroblasts, smooth muscle endothelial cells), and F4/80+ (macrophages). There was a reduction in the number of vascular cells that expressed CD31/PECAM, which we interpreted as reflective of the loss of endothelial cells with vascular rarefaction.

There were increased mRNA levels of the profibrotic cytokine transforming growth factor (TGF)- $\beta_{1}$, collagen type $1 \alpha_{1}$, and fibronectin. Kidney injury molecule- 1 protein levels were elevated in both kidney tissue and urine of animals injected thrice with DT when compared to controls or animals treated only once with DT. Thus, isolated primary injury to the renal epithelium triggers a graded inflammatory response, which depends on the characteristics of the injury, including whether it is recurrent. Although endothelial cells were not subject to the primary insult associated with DT administration, renal endothelial cells became activated and vascular rarefaction ensued. Inflammation is a well-known feature of AKI [11]. We believe the enhanced sequelae associated with maladaptive repair after repeated injury reflect pathophysiological processes that are operant in man. It is important to remember that the mouse is likely much more reflective of the young human with a great deal of renal reserve. Most of our patients who develop AKI, however, are elderly and already have a compromised kidney reserve and/or function. Thus, a single insult in humans, particularly if it is severe, can be followed by a maladaptive response, as seen in the multiple injury model in the mouse, including further development of glomerulosclerosis over time. Thus, recurrent, specific, tubular injury in a normal kidney, or less injury in a previously injured kidney, leads to a process of progression typical of CKD in humans. Clearly, the progressive renal disease has implications for the predisposition to the need for end-stage renal disease care, but CKD itself in the absence of ESRD also has marked effects on cardiovascular morbidity and mortality [12].

In the mouse, there was no initial glomerular injury associated with the administration of DT at a time when electron microscopy revealed dramatic injury to the tubule [10]. By 5 weeks in the thrice-administered group, however, there was focal global and segmental glomerulosclerosis. The degree of glomerulosclerosis was positively correlated with the degree of interstitial fibrosis/ tubular atrophy and amount of albuminuria. A progressive tubulointerstitial reaction originating around atrophic and undifferentiated tubules may compromise the vasculature and interrupt blood flow to the glomerulus 
causing secondary sclerosis. Alternatively, or in addition, there may be direct encroachment on the glomerular tuft due to the progressive fibrosis. Another contributor to the glomerulosclerosis could relate to the close proximity of early proximal tubular segments to the glomerular tuft. Paracrine signaling from injured and regenerating/undifferentiated epithelium may affect the glomerulus. In addition, damage to the tubule may decrease salt and water reabsorption, and the increased delivery to the distal nephron could then trigger the production of local vasoconstrictors that would reduce blood flow further [13]. Ultimately, the glomerulotubular junction may, in some nephrons, become progressively narrower and disconnect the glomerulus from the tubule [14].

\section{How Does the Inability of the Proximal Tubule Cell to Progress through the Cell Cycle Result in Maladaptive Repair and Fibrosis?}

Severe injury to the tubule may leave some of the cells in a dedifferentiated state for prolonged periods of time. These dedifferentiated cells may produce bioactive molecules with autocrine and paracrine functions, which may have detrimental effects and potentiate fibrosis $[4,15,16]$. De Borst et al. [17] reported that c-Jun $\mathrm{NH}_{2}$-terminal kinase (JNK) remained active in proximal tubule cells weeks after the ischemic insult and was associated with tubulointerstitial inflammation and fibrosis.

In order to explore features of repair that can lead to fibrosis, we compared the repair characteristics in a number of injury models, which represented different degrees of insult and etiology in the mouse [3]. Three models involved bilateral kidney injury: moderate ischemia, severe ischemia, and aristolochic acid nephropathy. These models were complemented with two unilateral models: unilateral ischemia and unilateral ureteral obstruction. In all cases, except for the moderate ischemia model, severe fibrosis developed (visualized by Masson's trichrome staining, collagen type 1 and 4 accumulation and biochemical determination of collagen) over time following the insult.

The cell cycle distribution in vivo during injury and recovery was characterized as a function of time in each of the models. We compared chromatin characteristics in vivo with chromatin patterns at various stages of the cell cycle in vitro. Proliferating cells (G1, S, G2 and M phase) were labeled in vivo with $K_{\mathrm{i}}-67$ specific antibody [18]. Sphase cells were identified by bromodeoxyuridine uptake [18]. Cells in the G2/M phase were determined by quantifying tubular cells with phosphorylation of histone $\mathrm{H} 3$ at

Maladaptive Proximal Tubule Repair: Cell Cycle Arrest
Ser10 by staining with a phosphorylation-specific antibody [19]. G2/M-arrested proximal tubular cells activated JNK signaling, resulting in upregulation of the production of profibrotic cytokines. Treatment with a JNK inhibitor, bypassing the $\mathrm{G} 2 / \mathrm{M}$ arrest by administration of a p53 inhibitor in the bilateral models or removing the contralateral kidney after unilateral ischemia, reduced fibrosis. Studies in vivo were complemented with studies on proximal tubule cells in vitro. When G0/G1 cells were separated from G2/M cells and analyzed, it was confirmed that the $\mathrm{G} 2 / \mathrm{M}$ cells generated much more proinflammatory and profibrotic cytokines and growth factors. TGF- $\beta$ and connective tissue growth factor featured prominently in these studies as mediators of the profibrotic re-sponse [3].

In rodents, other investigators have also subsequently demonstrated the therapeutic potential of blocking the initiation of the G2/M checkpoint, or encouraging cells to transit through G2/M to complete mitosis, via the use of agents such as histone deacetylase inhibitors [20] or p53 inhibition [21]. Biomarkers of cell cycle arrest have been identified in the urine of AKI patients [22].

\section{Conclusion}

We have developed a new, more targeted model of acute tubular injury to study pathophysiological mechanisms and outcomes in the kidney after isolated injury to renal proximal epithelial cells. Repeated injury resulted in maladaptive repair, resulting in prolonged inflammation, tubulointerstitial fibrosis, tubular atrophy, and secondary glomerulosclerosis. G2/M arrest in proximal tubule cells after kidney injury results in abnormal amplification of profibrogenic factors. These studies provide mechanistic links between AKI and chronic progressive fibrotic kidney disease. It is also of note that aging is associated with a decreased capacity to repair and regenerate injured tissue, including the kidneys $[23,24]$. There are many analogies that can be drawn between aging, senescence, and G2/M arrest which may result in a synthesis that is important for the understanding of the effects of aging on predisposition to CKD, the understanding of why patients with AKI are more prone to develop CKD, and why patients with $\mathrm{CKD}$ are predisposed to the development of AKI.

\section{Acknowledgment}

The work was supported by US National Institutes of Health (NIH) grants DK39773 and DK72381 to J.V.B.

Nephron Clin Pract 2014;127:61-64 


\section{References}

$>1$ Coca SG, Yusuf B, Shlipak MG, Garg AX, Parikh CR: Long-term risk of mortality and other adverse outcomes after acute kidney injury: a systematic review and meta-analysis. Am J Kidney Dis 2009;53:961-973.

$\checkmark 2$ Hsu CY: Yes, AKI truly leads to CKD. J Am Soc Nephrol 2012;23:967-969.

-3 Yang L, Besschetnova TY, Brooks CR, Shah $\mathrm{JV}$, Bonventre JV: Epithelial cell cycle arrest in G2/M mediates kidney fibrosis after injury. Nat Med 2010;16:535-543.

4 Venkatachalam MA, Griffin KA, Lan R, Geng H, Saikumar P, Bidani AK: Acute kidney injury: a springboard for progression in chronic kidney disease. Am J Physiol Renal Physiol 2010;298:F1078-F1094.

5 Bonventre JV, Yang L: Cellular pathophysiology of ischemic acute kidney injury. J Clin Invest 2011;121:4210-4221.

$\checkmark 6$ Basile DP, Donohoe D, Roethe K, Osborn JL: Renal ischemic injury results in permanent damage to peritubular capillaries and influences long-term function. Am J Physiol Renal Physiol 2001;281:F887-F899.

7 Bonventre JV: Kidney ischemic preconditioning. Curr Opin Nephrol Hypertens 2002 11:43-48.

$>8$ Park KM, Byun JY, Kramers C, Kim JI, Huang $\mathrm{PL}$, Bonventre JV: Inducible nitric oxide synthase is an important contributor to prolonged protective effects of ischemic preconditioning in the mouse kidney. J Biol Chem 2003;278:27256-27266.
9 Meyrier A, Hill GS, Simon P: Ischemic renal diseases: new insights into old entities. Kidney Int 1998;54:2-13.

10 Grgic I, Campanholle G, Bijol V, et al: Targeted proximal tubule injury triggers interstitial fibrosis and glomerulosclerosis. Kidney Int 2012;82:172-183.

11 Bonventre JV, Zuk A: Ischemic acute renal failure: an inflammatory disease? Kidney Int 2004;66:480-485.

12 Chawla LS, Amdur RL, Shaw AD, Faselis C, Palant CE, Kimmel PL: Association between $\mathrm{AKI}$ and long-term renal and cardiovascular outcomes in United States veterans. Clin J Am Soc Nephrol 2014;9:448-456.

13 Thomson SC, Blantz RC: Glomerulotubular balance, tubuloglomerular feedback, and salt homeostasis. J Am Soc Nephrol 2008;19: 2272-2275.

14 Chevalier RL, Forbes MS: Generation and evolution of atubular glomeruli in the progression of renal disorders. J Am Soc Nephrol 2008;19:197-206.

15 Suzuki T, Kimura M, Asano M, Fujigaki Y, Hishida A: Role of atrophic tubules in development of interstitial fibrosis in microembolism-induced renal failure in rat. Am J Pathol 2001;158:75-85.

16 Kimura M, Asano M, Abe K, Miyazaki M, Suzuki T, Hishida A: Role of atrophic changes in proximal tubular cells in the peritubular deposition of type IV collagen in a rat renal ablation model. Nephrol Dial Transplant 2005;20: 1559-1565.
17 de Borst MH, Prakash J, Sandovici M, et al: cJun NH2-terminal kinase is crucially involved in renal tubulo-interstitial inflammation. J Pharmacol Exp Ther 2009;331:896-905.

18 Yu CC, Woods AL, Levison DA: The assessment of cellular proliferation by immunohistochemistry: a review of currently available methods and their applications. Histochem J 1992;24:121-131.

19 Crosio C, Fimia GM, Loury R, et al: Mitotic phosphorylation of histone $\mathrm{H} 3$ : spatio-temporal regulation by mammalian Aurora kinases. Mol Cell Biol 2002;22:874-885.

20 Cianciolo Cosentino C, Skrypnyk NI, Brilli $\mathrm{LL}$, et al: Histone deacetylase inhibitor enhances recovery after AKI. J Am Soc Nephrol 2013;24:943-953.

21 Zhou L, Fu P, Huang XR, Liu F, Lai KN, Lan HY: Activation of p53 promotes renal injury in acute aristolochic acid nephropathy. J Am Soc Nephrol 2010;21:31-41.

$\checkmark 22$ Kashani K, Al-Khafaji A, Ardiles T, et al: Discovery and validation of cell cycle arrest biomarkers in human acute kidney injury. Crit Care 2013;17:R25.

23 Westhoff JH, Schildhorn C, Jacobi C, et al Telomere shortening reduces regenerative capacity after acute kidney injury. J Am Soc Nephrol 2010;21:327-336.

24 Clements ME, Chaber CJ, Ledbetter SR, Zuk A: Increased cellular senescence and vascular rarefaction exacerbate the progression of kidney fibrosis in aged mice following transient ischemic injury. PLoS One 2013;8:e70464. 